\title{
Uso de la robótica educativa como medio para favorecer la creatividad en la educación no formal
}

\section{Use of educational robotics to promote creativity in non-formal education}

\author{
Paula Morales Almeida \\ Universidad de Las Palmas de Gran Canaria (España) \\ paula.morales@ulpgc.es
}

Recibido: $15 / 01 / 2021$

Aceptado: 02/11/2021

Publicado: 01/12/2021

\section{RESUMEN}

En la actualidad asistimos a cambios sociales y tecnológicos que requieren una restructuración del sistema educativo, donde no se prime solo la educación formal, sino también la no formal, la que se lleva a cabo en el tiempo libre. Los menores, en estos ámbitos, también desarrollan habilidades y capacidades que les ayudan a mejorar sus competencias y a prepararse para el futuro. Una de las herramientas que se encuentra en auge para desarrollar las competencias básicas en los menores y, sobre todo, la competencia digital, es la robótica educativa, entendida esta como un proceso creativo basado en el ensayo-error, donde se utiliza la tecnología para interaccionar entre la sociedad, la ciencia y la propia tecnología, a través de la construcción, manipulación y programación de robots. En esta investigación se pretende aportar evidencias

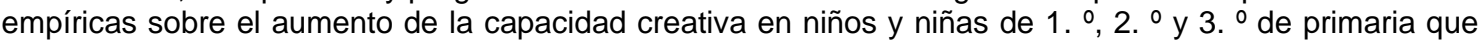
participan en un proyecto no formal, de ocio y tiempo libre. Para ello, se llevó a cabo un taller de robótica educativa donde los menores construyeron y programaron sus robots, con el programa WeDo 2.0, de Lego Education. Para comprobar si había habido cambios en la creatividad de los participantes se utilizó la prueba CREA, antes y después del taller. Los resultados obtenidos muestran un aumento significativo de la creatividad en los menores, aunque, debido a la pequeña muestra de este trabajo no podemos generalizarlo para el resto de la población.

\section{PALABRAS CLAVE}

Robótica educativa; creatividad; educación no formal; competencia digital; Lego Education.

\begin{abstract}
Nowadays we are witnessing social and technological changes that require a restructuring of the educational system, where not only formal education prevails, but also non-formal education, which is carried out in free time. Children in these areas also develop skills and abilities that help them improve their skills and prepare for the future. One of the tools that is booming to develop basic skills in minors and, above all, digital competence, is educational robotics, understood as a creative process based on trial-and-error, where technology is used to interact between society, science and technology itself, through the construction, manipulation and programming of robots. This research aims to provide empirical evidence on the increase in creative capacity in boys and girls in 1st, 2nd and 3rd grade of primary school who participate in a nonformal project of leisure and free time. For this, an educational robotics workshop was held where the children built and programmed their robots, with the WeDo 2.0 program, from Lego Education. To check if there had been changes in the creativity of the participants, the CREA test was used before and after the workshop. The results obtained show a significant increase in creativity in minors.
\end{abstract}

\section{KEYWORDS}

Educational robotics; creativity; informal education; digital competence; Lego Education. 


\section{CITA RECOMENDADA}

Morales, P. (2021). Uso de la robótica educativa como medio para favorecer la creatividad en la educación no formal. RIITE Revista Interuniversitaria de Investigación en Tecnología Educativa, 11, 85-97. https://doi.org/10.6018/riite.463631

Principales aportaciones del artículo y futuras líneas de investigación:

- Importancia de la educación no formal como favorecedora de la creatividad en menores.

- Utilización de la robótica educativa para fomentar habilidades y capacidades en los menores del siglo XXI.

\section{INTRODUCCIÓN}

Actualmente asistimos a cambios sociales y tecnológicos que hacen necesaria una restructuración del sistema educativo español y, con ello, plantear nuevos modelos de enseñanza-aprendizaje. Entre esos cambios encontramos la incorporación de las tecnologías de la información y la comunicación (TIC) en las aulas y la priorización de la competencia digital, dentro de las ocho competencias a trabajar, en la nueva Ley Orgánica de Educación (LOMLOE) (EURYDICE, 2021). Entre las modificaciones que ha ido sufriendo el sistema educativo español encontramos el "desarrollo de un nuevo modelo de currículo acorde con la adquisición de competencias, que sea abierto, flexible, con una estructura manejable y mejor integrada, al servicio de una educación inclusiva y que valore la diversidad" (LOMLOE, 2020, p.4). El alumnado del siglo XXI debe ser capaz de resolver problemas, de manera creativa y adquirir competencias científico-tecnológicas ante los retos que se le presentarán en un futuro, no muy lejano. Son ellos y ellas quienes se convierten en los y las protagonistas de sus propios procesos de enseñanza-aprendizaje, por lo que deben aprender a discernir la información que van obteniendo e incorporarlos a sus esquemas de conocimientos y saber cuándo deben utilizarlos, por tanto, se busca un aprendizaje significativo. La educación formal presta sus capacidades y recursos para ello, pero no es suficiente. Los menores, aparte de asistir a su centro educativo se desenvuelven en sus comunidades, se desarrollan en sus familias, con sus vecinos, en el centro educativo, acuden a actividades de educación no formal, clubes deportivos, actividades extraescolares... por lo que esta educación se erige como una formación igual de esencial y necesaria que la formal y debe proveer a los menores de herramientas y habilidades que les ayuden en su futuro profesional y personal.

La finalidad de este estudio es analizar y mejorar la creatividad de un grupo de niños con graves carencias familiares y sociales, a partir de un taller pedagógico de robótica educativa, utilizando para ello un diseño pre-test y post-test. Dicho taller se desarrolla en el espacio que los niños tienen para realizar actividades de ocio y tiempo libre, utilizando para ello un espacio no formal. Se ha llevado a cabo un estudio de tipo cualitativo pre-experimental, que consiste en llevar a cabo un pretest y un postest sin grupo de control. Lo situamos dentro del enfoque de un estudio de caso, por lo tanto, podemos decir que es descriptivo, inductivo y nos permite conocer una realidad singular.

\subsection{Robótica educativa}

Una de las nuevas herramientas pedagógicas ante las que nos encontramos que capacita al alumnado en competencias y habilidades propias del S.XXI, es la robótica educativa. Esta se ha ido expandiendo en los diversos ámbitos de la vida diaria y ya forma parte de la sociedad, tal y como afirman García et al. (2012). La educación también se ha visto transformada por esta herramienta, que busca ayudar a facilitar el conocimiento y el aprendizaje del alumnado. Como aseguran Cabello \& Carrera (2017):

La introducción de la robótica en las escuelas parece ser una buena estrategia para trabajar especialmente la creatividad, la experimentación, el trabajo en equipo y el 
aprendizaje a partir del error, elementos que pueden incidir en la mejora de los resultados académicos del alumnado (p.2).

En este nuevo sistema de aprendizaje, el estudiantado se convierte en el actor principal y el profesorado adquiere un rol de facilitador, de guía (Papadakis et al. 2016). El alumnado adquiere un papel más activo en su proceso de enseñanza-aprendizaje, ya que se erige como el creador de su conocimiento (Sánchez \& Guzmán, 2012). Estudios recientes aseguran que es posible alcanzar beneficios educativos con la robótica educativa, ya que facilita el aprendizaje del estudiante, a través de la investigación y de la experimentación y contribuye al desarrollo del conocimiento de las áreas STEM y se refuerza la creatividad, comunicación, cooperación y trabajo en equipo (Caballero \& García, 2020).

La escuela se erige como una de las protagonistas a la hora de preparar a los alumnos y alumnas para estos nuevos retos que presenta esta nueva sociedad, con lo cual, la integración de las TIC en la escuela es vital para el desarrollo integral del estudiante de educación primaria, por lo que debe buscar estrategias y metodologías novedosas e innovadoras que le ayuden en esta tarea. Así, pasaremos de "tener" el conocimiento a gestionarlo y de las TIC a las tecnologías del aprendizaje y conocimiento (TAC) (Vivas \& Sáez, 2019). Lo importante no será tener la información sino aprender a gestionarla y procesar la que es necesaria para convertirla en conocimiento a través de la tecnología. Si se consiguiera la introducción de las TIC y la robótica en concreto en el currículo se contribuiría a cerrar la brecha digital, mejorar la fluidez tecnológica de las personas y desarrollar el pensamiento computacional, tal y como afirman Martínez y Gómez (2018). Como afirma Pina (2017), "la formación en programación a nivel de primaria es una necesidad muy importante hoy en día" (p.17). Es una necesidad imperiosa por el tipo de sociedad digital en las que nos encontramos hoy en día que necesitará de trabajadores y trabajadoras formados en competencia digital para dar respuesta a los nuevos retos a lo que se enfrenta la población.

Pero, conviene recordar que la escuela no es la única protagonista ni tiene la exclusividad en cuanto a educación se refiere. La educación no formal, la que se desarrolla en el tiempo libre también ayuda a la adquisición de competencias, dado que este modelo también las promueve fuera del ámbito curricular, pero, como afirma Ruiz (2012), la educación no formal está infrautilizada e infravalorada. Esta educación también da respuesta a los problemas con los que se encuentra la sociedad hoy en día, por lo que debe innovar sobre nuevos métodos para promover una educación de calidad, en el tiempo libre, a través de metodologías innovadoras y el uso de la tecnología educativa (Cañellas, 2005).

Es frecuente ver, como, en los últimos tiempos, cada vez instituciones de educación no formal promueven actividades de robótica educativa, ya sea dentro o fuera de las aulas de educación formal (Bravo \& Forero, 2012). Suelen ser, la mayoría de ellos, proyectos liderados por empresas privadas (Pittí et al. 2014). Estos trabajos de robótica educativa implican el uso de motores, sensores y conectores, junto a otros elementos, que, unidos a la programación "dan vida" a los robots. Unos de los materiales más utilizados por estos grupos son los creados por Lego Education (Karp \& Maloney, 2013). Entre los diversos materiales creados por esta empresa encontramos el WeDo 2.0, utilizado en esta investigación. Este kit de robótica ha sido diseñado para estudiantes de educación primaria, cuyas edades oscilan entre los 6 a 11 años, permitiéndoles "diseñar y poner en actividad prototipos robóticos a través de determinados softwares que le permiten desarrollar competencias, capacidades y habilidades relacionadas con la tecnología" (Cisneros, 2015, p.82).

Como afirman Zarza \& Holgado (2020), el uso de la robótica educativa favorece los procesos de enseñanza-aprendizaje en la educación en el tiempo libre, de una manera lúdica y en un ambiente cordial, pero, el principal problema de estas actividades suele consistir en que no se extiende a todos los menores, ya sea por su elevada implicación económica y por la dificultad que pueden tener varias familias si no pueden afrontar los gastos de la actividad y esta no está subvencionada con fondos públicos (Vega et al. 2016). Otras investigaciones internacionales (Cakir et al., 2021; Yusuf, 2019; Leyla \& Ferhat, 2020) señalan que el uso de la robótica educativa ha ayudado a que el alumnado sea más flexible, fluido, imaginativo, que se adapte mejor a las circunstancias, por lo que abogan por su uso.

La robótica educativa es una herramienta que se puede integrar perfectamente en un entorno de aprendizaje STEAM que tiene mucho que aportar a la motivación, interés y rendimiento de los estudiantes (Ruiz, 2017). 


\subsection{La creatividad desarrollada a través de la robótica educativa}

Entre las muchas capacidades y habilidades que la robótica educativa puede desarrollar en los menores, destacamos especialmente una por encontrarse dentro de nuestra investigación, la creatividad. En palabras de Corbalán \& Limiñana (2010):

La creatividad representa para la humanidad, la dimensión psicológica en que se ha fundado su vertiginosa evolución como especie que, más allá de su conformación biológica, ha generado una transformación cultural que la hace reinventarse de continuo en cuanto a sus formas de vida, su conocimiento del cosmos y su conocimiento de sí misma (p.197).

Así, aunque la creatividad siempre ha estado ahí, desde los albores de la humanidad, es, desde hace pocos años, tenida en cuenta y reconocida socialmente. No se encuentra tampoco en el currículo escolar, por lo que muchos niños, creativos, han quedado fuera del sistema porque este no ha sabido tratar su inteligencia creativa. Asistimos actualmente a un reconocimiento de la creatividad y de la necesidad de contar con ella para poder responder, de otra manera, a los problemas con los que nos enfrentaremos en un futuro cercano (Corbalán \& Limiñana, 2010). Aun así, muchos autores reconocen que la evaluación de la creatividad no es una empresa sencilla, puesto que hay que tener en cuenta múltiples factores que la condicionan (Donolo \& Elisondo, 2007). Supone también, sortear una serie de obstáculos y aceptar ciertos desafíos.

Pero ¿cómo se define la creatividad? Desde los comienzos del término se delimitó como la habilidad que una persona posee para resolver preguntas y enfrentarse a problemas (Smilansky \& Halberstadt, 1986), teniendo en cuenta que una persona es creativa cuando es capaz de enfrentarse a retos y superarlos, incluso dando una respuesta diferente a la que dan otras personas que no tienen tan desarrollada la creatividad. Otros autores señalan que es una habilidad intelectual (Hu \& Adey, 2002) o que es generadora de ideas nuevas (Sarmiento, 2017). En lo que concuerdan la mayoría de ellos es en señalar que la persona creativa es flexible, da respuestas originales ante diversas situaciones y elabora sus propias ideas.

A pesar de la dificultad de definir este término y por el que los autores no se ponen de acuerdo, sí se puede afirmar que la creatividad es una habilidad con la que no se nace, sino que se puede entrenar. El hecho de que haya personas más creativas que otras no se debe a un rasgo de personalidad, sino que hay unas personas con más facilidad para el pensamiento lateral que otras, lo que no significa que no se pueda entrenar y mejorar en esta habilidad.

La robótica educativa fomenta en el niño y la niña la toma de decisiones, a la hora de programar el robot para que realice una determinada función y le hace responder de forma creativa a ciertos problemas, buscando otras maneras de llegar a una misma solución (Vivas \& Saéz, 2019). Es necesario que se fomente este tipo de actividades no solo en la educación formal, sino también en la no formal, ya que los niños, en esos entornos, aprenden de una manera más significativa y en un ambiente distendido.

\section{MÉTODO}

Los objetivos de esta experiencia son:

- Analizar y mejorar la creatividad de un grupo de niños y niñas con graves carencias familiares y sociales.

- Fomentar el uso de la robótica educativa en el ámbito no formal.

- Valorar el uso que la robótica educativa puede proporcionar al aumento y desarrollo de la creatividad.

Esta experiencia se ha llevado a cabo a través de un estudio de tipo cualitativo pre-experimental, en el que existen un pretest y un postest, sin grupo de control. Se sitúa dentro del enfoque de estudio de casos, es un método de investigación que implica una actividad de indagación de una realidad concreta. Por otra parte, el muestreo es no probabilístico e intencional, por lo que no se podrán generalizar los datos obtenidos para el resto de la población. La muestra la conforman 21 personas $(n=21)$. 


\subsection{Participantes}

En esta investigación participaron 21 menores $(n=21)$, de entre 6 y 9 años. De los 21 participantes, 14 son niñas y 7 son niños. 8 de ellos cursan 1.0 de primaria, 4 ; 2 - de primaria y los 9 restantes, 3. de primaria, en el momento de la realización de la investigación. Estos 21 niños pertenecen a un proyecto de infancia llamado Media Luna, donde realizan actividades socioeducativas en su ocio y tiempo libre, ya que pertenecen a familias desestructuradas, por lo que no tienen oportunidad de participar en otros espacios formativos o educativos y en sus centros educativos de referencia no cuentan con programas de robótica educativa, dado que la zona en la que viven, Jinámar, es un barrio que se encuentra entre las dos ciudades más grandes de la Isla de Gran Canaria; Las Palmas de Gran Canaria y Telde y cuenta con muchos problemas de índole social. Sus familias no pueden costearse ningún tipo de curso en el tiempo libre de los menores, por ello, acuden a este proyecto, donde no tienen que costear las actividades, puesto que está financiado por las administraciones locales. Así, los menores, tienen un espacio donde poder seguir formándose y alcanzar habilidades y capacidades que no les dejen en desventaja con respecto a otros niños y niñas de otras zonas. Por ello, centramos esta investigación en la educación no formal, dotándola de la importancia que tiene para desarrollar otro tipo de habilidades y capacidades en los menores, aparte de la educación formal.

\subsection{Instrumentos}

El material empleado para realizar el taller de robótica educativa con los 21 niños fue el WeDo 2.0, un material de Lego Education. Este robot fue creado en 2016 por la empresa Lego Education, enfocado a niños de edades comprendidas entre los seis y los once años. Trabaja con un software basado en programación visual por iconos desarrollado por la misma empresa. En la figura 1 podemos ver una imagen del robot.

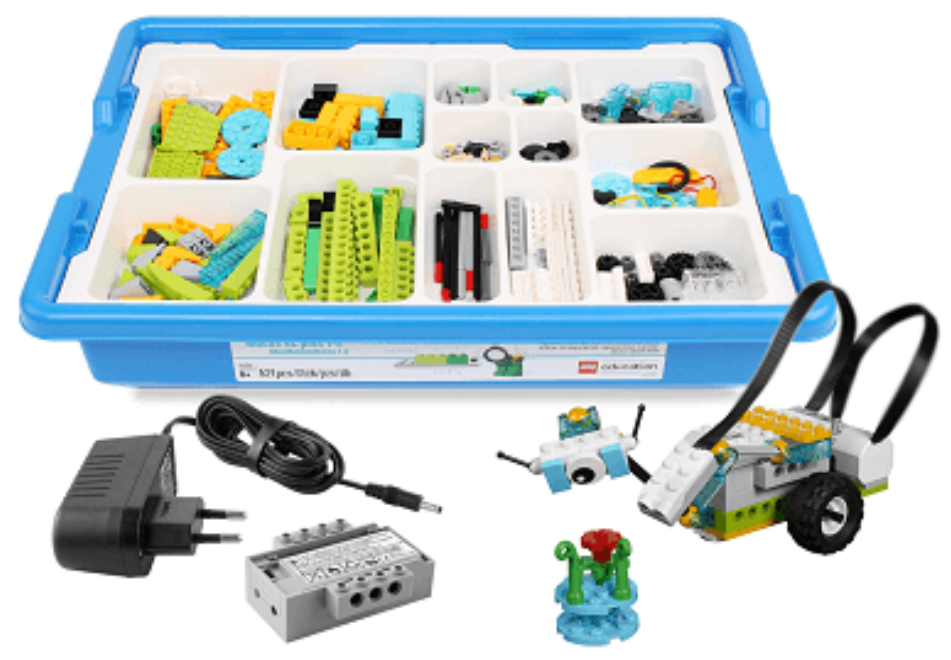

Figura 1: Robot WeDo 2.0. Fuente: Ciencia y Robótica (2021).

Otro material empleado e igual de necesario fue la prueba de creatividad CREA (Corbalán et al. 2015) realizada a los menores, antes y después del taller. "Este instrumento permite acceder a una única medida de la capacidad creativa de los sujetos. Está relacionada con los factores tradicionalmente utilizados para definir la creatividad: originalidad, fluidez, flexibilidad y producción divergente" (Donolo \& Elisondo, 2007, p. 148). EI CREA utiliza la capacidad de elaboración de preguntas del sujeto, como medida de la creatividad. Nos permite conocer la capacidad creativa de las personas a través de una prueba cognitiva individual de su creatividad, donde tienen que hacerse preguntas a través de la visualización de una imagen. La aplicación de la prueba consiste en la presentación de 1 lámina, la $C$, ante la que los menores tienen que hacerse el mayor número de preguntas posible. Se presenta esta lámina dado que es la que se utiliza para la población entre 6 y 9 años.

Esta prueba tiene un coeficiente Alpha de Cronbach de 0,775 , por lo que podemos determinar la alta fiabilidad de esta. Para conocer la puntuación de los menores, López \& Martín (2010) comentan que: 
El CREA proporciona una puntuación directa (PD) que debe ser consultada en los baremos correspondientes para obtener una puntuación centil (PC) que posteriormente es interpretada. A tal fin los autores presentan una tabla de criterios interpretativos generales sobre las características creativas de sujetos, basados en los percentiles y agrupados según la puntuación obtenida en la prueba respectiva como creatividad alta, media y baja. (p.256)

La administración del instrumento y la corrección de los protocolos se realizaron conforme a las pautas del manual correspondiente. Se administró el instrumento de manera individual y se utilizó la lámina estímulo $\mathrm{C}$ para todos los menores, como se comentó anteriormente. En la figura 2 podemos observar la lámina $\mathrm{C}$.

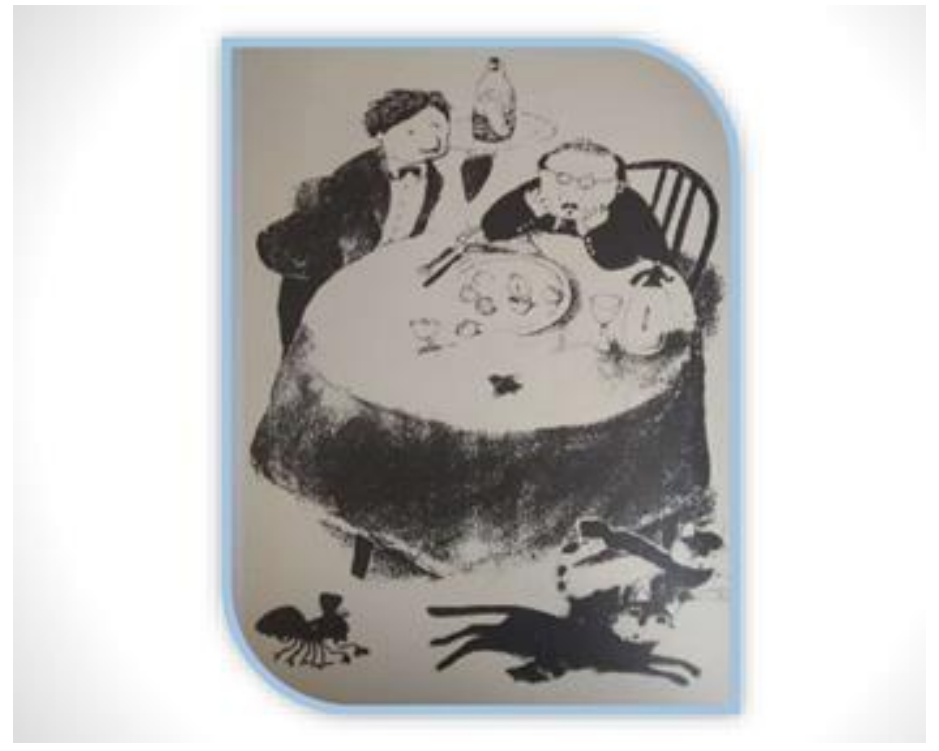

Figura 2. Lámina C del CREA. Fuente: Corbalán et al. (2015).

Para realizar esta prueba, se le pide al menor que formule cuantas preguntas se le ocurran sobre la imagen en cuestión. Al terminar el tiempo, 4 minutos, se contabilizan las preguntas realizadas. Para corregir el test contamos cuántas preguntas ha realizado el menor anotando doble y triple a aquellas que contengan varias preguntas en sí mismas. Se anotan las preguntas realizadas $(\mathrm{N})$, los espacios vacíos $u$ omisiones $(\mathrm{O})$, las preguntas anuladas $(\mathrm{An})$ y la puntación extra (Ex). Finalmente se hace el cálculo final aplicado esta fórmula: $P D=N-O-A n+E x$. La puntuación directa obtenida (PD) se consulta en los baremos correspondientes para obtener la puntuación centil (PC), que pasa a ser interpretable.

\subsection{Procedimiento}

El taller tuvo una duración de 8 horas divididas en cuatro semanas. Se realizaron 2 sesiones en una semana, de 1 hora cada una. Antes del comienzo del taller se rellenaron todos los pretest y el último día, se cumplimentaron los postest, por lo que, en total, se dedicó 10 horas al taller. La primera sesión fue de conocimiento del material y cómo íbamos a trabajar y las siguientes sesiones se centraron en la construcción y programación de los diferentes robots. En la tabla 1 se puede observar las sesiones llevadas a cabo. 
Tabla 1. Distribución de las sesiones del taller

\begin{tabular}{cllr}
\hline $\begin{array}{c}\text { No } \\
\text { Sesión }\end{array}$ & \multicolumn{1}{c}{ Sesión } & \multicolumn{1}{c}{ Material } & Duración \\
\hline $\mathbf{0}$ & $\begin{array}{l}\text { Presentación del programa y test } \\
\text { CREA }\end{array}$ & Test CREA & 1 hora \\
$\mathbf{1}$ & $\begin{array}{l}\text { Presentación del WeDo 2.0. } \\
\text { Construcciones y programaciones }\end{array}$ & $\begin{array}{l}7 \text { cajas WeDo 2.0 } \\
7 \text { tabletas }\end{array}$ & 1 hora \\
& libres & & \\
$\mathbf{2}$ & Construcción y programación manual & & \\
$\mathbf{3}$ & Construcción y programación manual & & \\
$\mathbf{4}$ & Construcción y programación manual & & \\
$\mathbf{5}$ & Construcción y programación manual & & \\
$\mathbf{6}$ & Carrera de vehículos & & \\
$\mathbf{7}$ & Construcciones libres y creativas & & \\
$\mathbf{8}$ & Construcciones libres y creativas & Test CREA & \\
$\mathbf{9}$ & Post test CREA & & \\
& &
\end{tabular}

Para poder llevar a cabo el taller, cada grupo, formado por 3 personas, contó con una caja de material de WeDo 2.0 y una tableta. En la tableta tienen el software instalado con el que pueden seguir las instrucciones para construir diferentes robots y programarlos. La idea es que el equipo trabaje cooperativamente, uno de ellos se encarga de pasar las imágenes en la tableta, otro busca las piezas y el tercero lo monta. Al cabo de poco tiempo, los roles cambian, para así propiciar que todos los menores asuman todos los roles. Con el paso de las sesiones, es lógico que algunos niños/as se queden con un rol en concreto, porque les gusta más o se sienten más cómodos.

La intervención se llevó a cabo en tres fases bien diferenciadas. Por un lado, primero tuvo lugar la fase de planificación, donde se mantuvo una reunión con las familias de los menores para contarles el taller que queríamos poner en marcha y pedir su autorización, al igual que a los voluntarios del proyecto. Muchas de las familias no acudieron, algunas por estar trabajando y otras porque no participan en las actividades de sus hijos, aun así, realizamos una segunda sesión porque nos interesaba que los menores no perdieran esta oportunidad. Finalmente, al saber que no debían pagar nada del taller, las familias accedieron a que sus hijos realizaran el taller, incluso, algunas de ellas preguntaron si podrían estar presentes en las sesiones, pues les interesaba el tema. A lo cual, desde el equipo de investigación, se accedió a que pudieran estar presentes. Creemos que, de esta manera, se refuerza el vínculo y la parentalidad positiva entre padres, madres e hijos/as.

La segunda fase fue la de desarrollo, donde pusimos en marcha el taller de robótica educativa que tuvo lugar durante todo un mes, dos sesiones a la semana de 1 hora cada sesión. En esta fase, los niños aprendieron a montar los robots y programarlos y se les pidieron una serie de retos que tenían que ir consiguiendo para poder valorar en qué medida iban conociendo el manejo del programa. Los retos eran muy diversos, desde algunos muy sencillos, como conseguir que el robot avanzara o retrocediera sobre sí mismo, hasta algunos más complicados, como elaborar todo un circuito. En la sesión 8, la última del taller, tuvo lugar una demostración entre todos los equipos sobre los retos llevados a cabo. La idea fue que los menores se motivaran sobre el trabajo que estaban llevando a cabo ese mes y lo compartieran con los demás grupos. Todos los equipos participaron, bajo un nombre y un lema.

Por último, se llevó a cabo la tercera fase, la de evaluación, donde se realizaron los test a los alumnos y luego se realizó el vaciado de datos para obtener los resultados y así, poder analizar si ha habido cambios en la inteligencia creativa con el desarrollo del taller de robótica educativa. En la figura 3 se puede observar un esquema de las fases implementadas. 


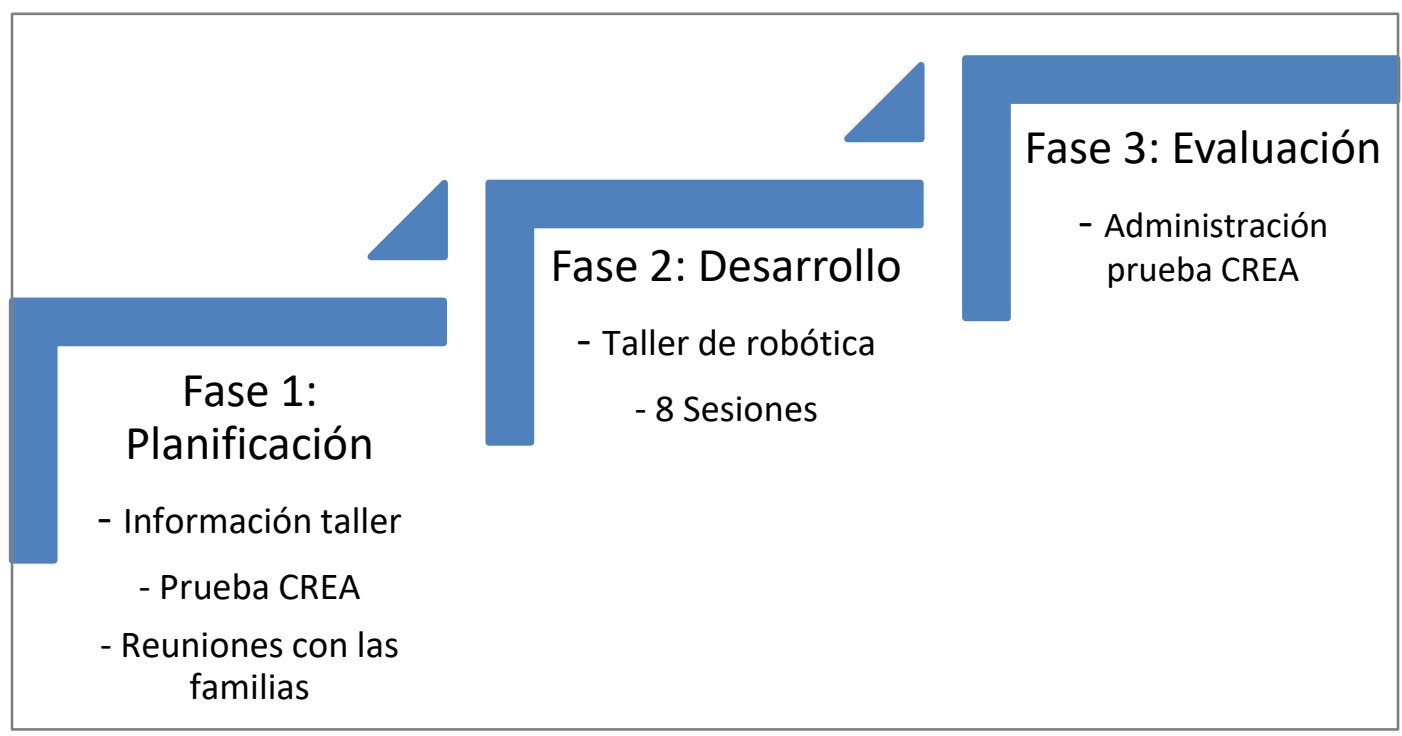

Figura 3. Implementación de las fases de la investigación

Tras la realización de las pruebas y el análisis de resultados, se mantuvo otra reunión con las familias para comentarles los avances que se había logrado con el taller y para que comprendieran la importancia de que sus hijos/as aprendan estas nuevas metodologías y conozcan estas herramientas de cara a su futuro. Ellas se mostraron de acuerdo con lo hablado en la reunión, pero también mostraban su preocupación por no ser capaces de costear todo esto para que sus hijos tuvieran un mejor acceso a la educación y un futuro mejor.

\section{RESULTADOS}

El análisis de los resultados es descriptivo. Se ha utilizado el programa IBM SPSS Statistics v.24. Además, se ha utilizado la prueba de Kolmogórov-Smirnov $(p=0,02)$ que determinó que la variable creatividad se distribuía adecuadamente. Así, para realizar la comparación de los resultados del pre y post test se decidió emplear esta prueba para comprobar si existían diferencias significativas en la creatividad antes y después de la realización del taller. Para los valores de la creatividad obtenidos en el pre y post test, el contraste de medias reveló diferencias estadísticamente significativas entre los resultados de los participantes.

En la lámina $C: t=-2,0151 p=0,0248$. Por lo que podemos hablar de valores normales dentro de la muestra y de que los datos obtenidos son válidos y pueden ser tenidos en cuenta. Estos datos, así como la media y la desviación típica pueden observase en la tabla 2.

En la lámina administrada a los menores, la $\mathrm{C}$, comprobamos cómo ha habido cambios significativos antes y después de realizar el taller. Lo que, al no existir grupo de control no podemos determinar que haya sido por el taller de robótica educativa, pero existe un cambio entre antes de haberse realizado y después.

También se ha podido observar diferencias entre las edades de los menores, siendo los más pequeños, los de 1ํ de primaria, donde se han observado más cambios tras la realización del taller. Los más pequeños han participado más activamente en el taller.

Respecto a la incidencia del sexo en el CREA, se observan diferencias estadísticamente significativas a favor de las niñas que podemos encontrar en la tabla 3. Quizás por el hecho de que fueron el doble de participantes o porque puedan estar más interesadas en este tipo de iniciativas. 
Tabla 2. Media y desviación típica de la administración del CREA

\begin{tabular}{lll}
\hline & & CREA \\
\hline$N$ & \\
$M$ & 21 \\
$D E$ & 7.82 \\
$T$ & 5.66 \\
$p$ & 2,0151 \\
& 0,0248 \\
\hline
\end{tabular}

Tabla 3. Diferencias por sexo

\begin{tabular}{ccc}
\hline Sexo & & CREA C \\
\hline Niñas & $M$ & 8.30 \\
& $D E$ & 5.60 \\
Niños & $M$ & 7.30 \\
& $D E$ & 5.68 \\
& $t$ & 2.68 \\
& $P$ & $\mathbf{. 0 0 7}$ \\
\hline
\end{tabular}

Aunque las pruebas se realizaron de manera individual, hemos realizado la media grupal para conocer el nivel de creatividad del grupo antes y después de la realización del taller. En la figura 4 se puede observar la puntuación centil obtenida por el grupo en el pretest y en el post test. Para entender la puntuación centil podemos aclarar que:

- Entre el centil 75 y el 99: Creatividad Alta

- Entre el centil 26 y el 74: Creatividad Media

- Entre el centil 1 y el 25: Creatividad Baja:

Con los datos obtenidos podemos decir que, los menores, antes de comenzar con el taller de WeDo 2.0 tenían una creatividad baja, en torno a un centil 24, mientras que, una vez acabado el taller, tras la administración de la prueba CREA, obtuvieron, de media, un centil 63, que corresponde a una creatividad alta.

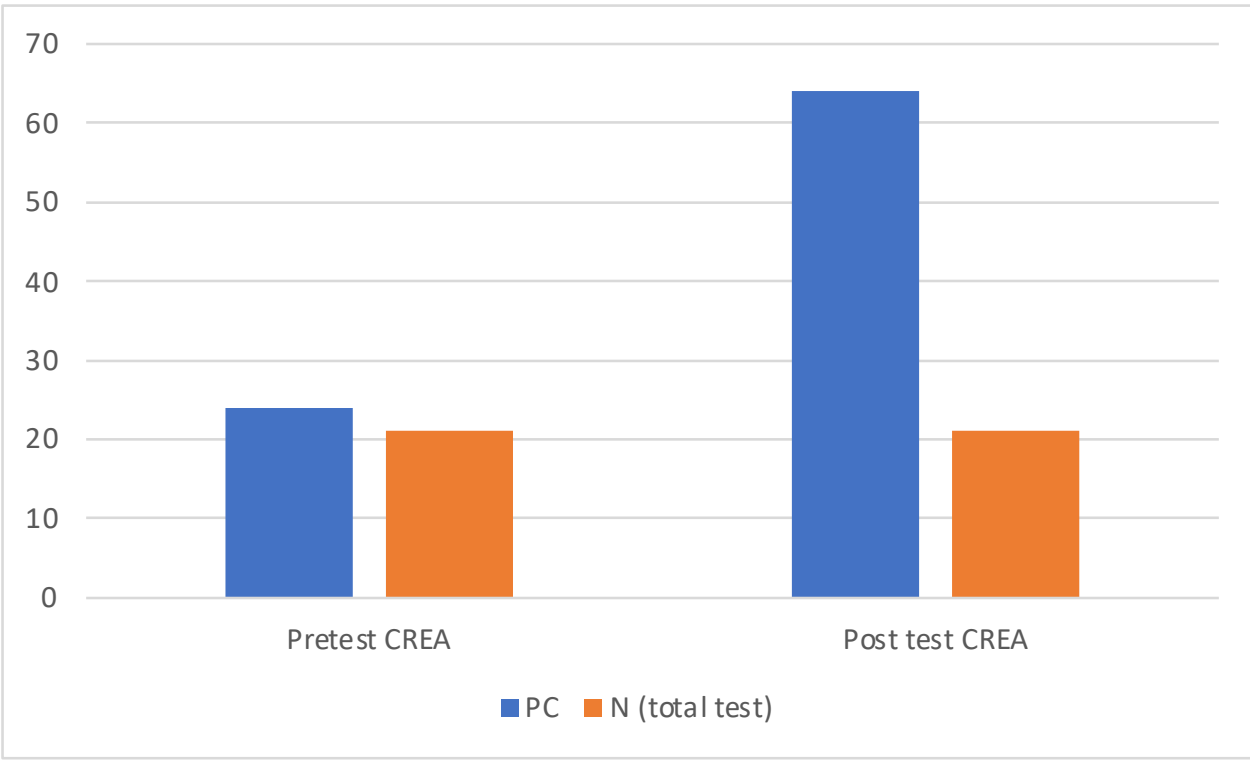

Figura 4. Media grupal de la prueba CREA. 
Los resultados obtenidos confirman que la realización del taller de robótica educativa supuso un aumento de la creatividad en los menores, al menos en los que realizaron el taller. No podemos afirmar que la creatividad alcanzada sea alta, pero es bastante más elevada de lo que era antes de la realización del taller, donde la creatividad de los menores se situaba en la parte baja. Los alumnos partícipes de esta investigación han visto incrementada su creatividad en la aplicación postest de la prueba CREA. Todo ello tras el trabajo realizado durante 10 sesiones con el programa de robótica WeDo 2.0 de Lego Education, donde tuvieron la oportunidad de trabajar en equipo, de construir robots, de programarlos. En una asamblea final, los menores comentaron lo contentos que estaban por haber podido participar en este tipo de proyectos y haber trabajado con un robot. Por tanto, se puede afirmar que la participación en el proyecto fue eficaz para incrementar la creatividad, tanto de forma global como en cada uno de los menores que participaron, además de propiciar otro tipo de habilidades y capacidades en los menores. Aún así, es necesario seguir trabajando en estos aspectos para conseguir que los menores alcancen una creatividad alta.

\section{DISCUSIÓN Y CONCLUSIONES}

Según algunos autores, la creatividad se entiende como la capacidad de dar respuestas o soluciones originales a los problemas (Waisburd, 2004). Pero, para que dicha respuesta se considere válida y reconocida en el entorno en el que se produce tendrá que ser adoptada y reconocida por el propio entorno. Así, no es suficiente con que la idea sea original, sino que tendrá, además, que ser útil (Acuña-Zúñiga, 2012). La educación debe favorecer el pensamiento creativo, para que los menores puedan dar respuestas originales a los diferentes problemas que se les vayan planteando. Y no solo es tarea de la educación formal, sino también de la no formal, dado que en esos entornos los niños aprenden también, e incluso más, de una manera más significativa, en la mayoría de las ocasiones. García (2015, p.3) analizó "la robótica como parte del proceso educativo, entendiendo que programar va más allá del acto de codificar, ya que conforma un conjunto de acciones que giran en torno a cuatro palabras clave: imaginar, diseñar, construir y programar". Con la robótica educativa, los menores pueden trabajar en equipo, de manera cooperativa, además, desarrolla en ellos las habilidades para resolver problemas, que no es otra cosa que fomentar su creatividad (Cakir et al. 2021). La robótica educativa, en cualquiera de sus programas y softwares propicia que los menores alcancen las competencias básicas y las habilidades propias del siglo XXI.

Con respecto a nuestra investigación, la realización de un taller con WeDo 2.0 ha permitido que exista un cambio en la creatividad en los 21 menores participantes, aunque no podemos afirmar que sea debido a ello, debido a la inexistencia de un grupo de control, pero existe una relación. Es necesario que se potencie este tipo de actividades, a través de la robótica educativa porque permite desarrollar habilidades y destrezas en los menores que les ayudarán como ciudadanos del S.XXI.

No podemos olvidar que hay familias que no pueden costearse estas actividades que no se encuentran aún presentes en el currículo de educación primaria, por lo que los menores de estas familias se ven en desventaja con respecto a los demás niños, porque sus familias se encuentran en situación de exclusión social y, con ello, se fomenta la brecha digital. De ahí que es necesario que las administraciones locales financien este tipo de talleres para aquellas familias que no pueden permitírselo y que así llegue a todos los niños, sin distinción de clase.

Es necesario potenciar y desarrollar la robótica educativa en educación, no solamente en las aulas de los centros educativos, sino también fuera de ellas, en los proyectos que trabajan con los menores en el ocio y tiempo libre, que sea de libre acceso y que les garantice una igualdad de oportunidades con las que poder enfrentar un mejor futuro (Sánchez \& Casallas, 2019).

Por otro lado, es necesario señalar las limitaciones de este estudio, dado que no ha existido un grupo de control y el tamaño de la muestra es bastante pequeño. Es necesario seguir investigando en este campo y llegar a más menores, contando con grupos de control y otros instrumentos de evaluación que permita conocer hasta qué punto la robótica educativa y la creatividad están o no relacionadas. 
Consideramos que la robótica educativa y el pensamiento computacional tienen mucho camino aún por andar, no solo dentro del currículo en la educación formal, sino en la no formal, llegando a todos los territorios y entornos favoreciendo la igualdad de oportunidades para que todos puedan participar. Como proyecto futuro se quiere llegar a más niños y niñas que puedan disfrutar con la robótica y ver cómo afecta a sus propias habilidades y capacidades, no solo a la creatividad.

La incorporación de herramientas educativas como la robótica, tanto dentro como fuera de las aulas, resulta muy beneficiosa para los menores, ya que favorece su capacidad creativa para la resolución de problemas. Pero, esta herramienta, por sí sola, no mejora el aprendizaje ni las capacidades del menor. Es necesario complementarla con otras herramientas y metodologías innovadoras que permitan el desarrollo integral del niño. Es por ello, que hay que seguir investigando en esta línea y conocer todo lo que la robótica educativa tiene aún por ofrecer.

\section{ENLACES}

Prueba CREA utilizada: https://bit.ly/3nXFcKm (se utilizó en formato papel, al igual que el material WeDo 2.0, que se utilizó de forma manipulativa, ya que se tiene el material físico).

\section{REFERENCIAS BIBLIOGRÁFICAS}

Acuña-Zúñiga, A. L. (2012). Diseño y administración de proyectos de robótica educativa: lecciones aprendidas. Teoría de la Educación. Educación y Cultura en la Sociedad de la Información, 13(3), 6-27. https://bit.ly/3pirUa2

Bravo, F.A. \& Forero A. (2012). La robótica como un recurso para facilitar el aprendizaje y desarrollo de competencias generales. TESI (Revista Teoría de la Educación. Educación y Cultura en la Sociedad de la Información), 13(2), 120-136. http://bit.ly/3rO4bAh

Caballero, Y. A. \& García, A. (2020). Fortaleciendo el pensamiento computacional y habilidades sociales mediante actividades de aprendizaje con robótica educativa en niveles escolares iniciales. Pixel-Bit. Revista de Medios y Educación, 58, 117-142. https://doi.org/10.12795/pixelbit.75059

Cabello, S. \& Carrera, X. (2017). Diseño y validación de un cuestionario para conocer las actitudes y creencias del profesorado de educación infantil y primaria sobre la introducción de la robótica educativa en el aula. EDUTEC, Revista Electrónica de Tecnología Educativa, 60. https://doi.org/10.21556/edutec.2017.60.871

Cakir, R., Korkmaz, O., Idil, O. \& Erodmus, F. (2021). The effect of robotic coding education on preschoolers' problem solving and creative thinking skills. Thinking Skills and Creativity, 40. https://bit.ly/3ojMfPq

Cañellas, A. (2005). Continuidad y complentariedad entre la educación formal y no formal. Revista de educación, 338, 9-22. https://bit.ly/2Zd33d6

Ciencia y robótica. (2021). Imagen de WeDo 2.0. https://bit.ly/3urMnNR

Cisneros, B. (2015). Uso y aplicación de las laptops XO y kit de robótica educativa WEDO en las Instituciones Educativas Públicas de Educación Primaria de la provincia de Tarma. [Tesis de maestría no publicada]. Universidad Femenina del Sagrado Corazón.

Comisión Europea (Ed.) (2012). El Desarrollo de las competencias clave en el contexto escolar en Europa: desafíos y oportunidades para la política en la materia. Informe de Eurydice. Oficina de Publicaciones de la Unión Europea. https://bit.ly/3tTHPzi

Corbalán, J., Martínez, F., Donolo, D., Alonso, C., Tejerina, M., \& Limiñana, M. R. (2015). CREA. Creative intelligence, a cognitive measure of creativity. TEA Editions. https://bit.ly/3dapTKG

Corbalán, J. \& Limiñana, R. (2010). El genio en una botella. El test CREA, las preguntas y la creatividad. Anales de psicología, 26(2), 197-205. http://bit.ly/3jJXvQW 
Donolo, S. \& Elisondo, R. (2007). Creatividad para todos. Consideraciones sobre un grupo particular. Anales de psicología, 23(1), 147-151. https://bit.ly/3aevyNV

EURYDICE. (2021). Reformas en educación primaria y secundaria. Comisión Europea. https://bit.ly/2Y2iTdd

García, J. M. (2015). Robótica educativa. La programación como parte de un proceso educativo. RED Revista de Educación a Distancia, 46(8), 1-11. https://bit.ly/3pgwBBp

García, M.N., Castillo, L.F. \& Escobar A.J. (2012). Plataforma robótica educativa "ROBI". Revista Colombiana de Tecnologías de Avanzada, 19(1), 140-144. https://doi.org/10.21556/edutec.2017.60.871

Hu, W. \& Adey, P. (2002). A scientific creativity test for secondary school students. International Journal of Science Education, 24(4), 389-403. https://doi.org/10.1080/09500690110098912

Karp, T., \& Maloney, P. (2013). Exciting Young Students in Grades K-8 about STEM through an Afterschool Robotics Challenge. American Journal of Engineering Education, 4(1), 39-54. https://doi.org/10.19030/ajee.v4i1.7857

Ley Orgánica 8/2013, de 9 de diciembre, para la Mejora de la Calidad Educativa (LOMCE). (2013). Boletín Oficial del Estado, 295. https://bit.ly/3tSzfAU

Ley Orgánica de 2/2006, de 3 de mayo, de Educación (LOE). (2006). Boletín Oficial del Estado, 106. http://bit.ly/3qgFMTI

Ley Orgánica 3/2020, de 29 de diciembre, por la que se modifica la Ley Orgánica 2/2006, de 3 de mayo, de Educación. Boletín Oficial del Estado, 340. https://bit.ly/3wpVqi1

Leyla, U. \& Ferhet, B. (2020). The Effect of Lego Wedo 2.0 Education on Academic Achievement and Attitudes and Computational Thinking Skills of Learners toward Science. World Journal of Education, 10(4). https://bit.ly/39P5yl2

López, O. \& Martín, R. (2010). Estilos de pensamiento y creatividad. Anales de psicología, 26(2), 254-258. http://bit.ly/3d3iexT

Martínez, M., \& Gómez, M. (2018). Programar computadoras en Educación Infantil. Edutec. Revista Electrónica de Tecnología Educativa, O(65), 40-53 (394). https://doi.org/10.21556/edutec.2018.65.1103

Papadakis, S., Kalogiannakis, M. \& Zaranis, N. (2016). Developing fundamental programming concepts and computational thinking with ScratchJr in preschool education: a case study. International Journal of Mobile Learning and Organization, 10(3), 187. https://doi.org/10.1504/ijmlo.2016.077867

Pina, A. (2017). Robótica Educativa en Educación Primaria: ¿por qué y cómo? En S. PérezAldeguer, G. Castellano-Pérez, y A. Pina-Calafi (Eds.), Propuestas de Innovación Educativa en la Sociedad de la Información (pp. 15-27). Adaya Press. https://bit.ly/3agrBhr

Pittí, K., Curto, B., Moreno, V. \& Rodríguez, M. J. (2014). Uso de la Robótica como herramienta de aprendizaje en Iberoamérica y España. VAEP-RITA, 2(1), 41-48. https://bit.ly/3daqqfE

Ruiz, F. (2017). Diseño de proyectos STEAM a partir del currículum actual de Educación Primaria utilizando Aprendizaje basado en problemas, aprendizaje cooperativo, flipped clasroom y robótica educativa. [Tesis doctoral, Universidad CEU Cardenal Herrera]. Alfara del Patriarca. https://bit.ly/3deSkae

Ruiz, R. (2012). L'educació en el lleure a l'inici del segle XXI a Catalunya. Educació social. Revista d'intervenció socioeducativa, (50), 69-79. http://bit.ly/3tMIGTv

Sánchez, F. Á. B. \& Guzmán, A. F. (2012). La robótica como un recurso para facilitar el aprendizaje y desarrollo de competencias generales. Education in the Knowledge Society, 13(2), 120-136. https://doi.org/10.14201/eks.9002 
Sánchez, B. \& Casallas, C. (2019). Desarrollo de habilidades STEM acercando el pensamiento computacional a niñas en situación de vulnerabilidad del municipio de Fusagasugá. (tesis doctoral). https://bit.ly/3okaRI2

Sarmiento, J. A. (2017). Desarrollo del pensamiento crítico y creativo mediante estrategias interconectadas: estrategias de aprendizaje, lectura crítica y ABP. Gestión, competitividad e innovación, 5(2), 145-162.

Smilansky, J., \& Halberstadt, N. (1986). Inventors versus problem solvers: An empirical investigation. Journal of Creative Behavior, 20(3), 183-201. http://dx.doi.org/10.1002/i.21626057.1986.tb00436.x

Vega, D., Cufíé, X., Rueda, Mạ . J., \& Llinás, D. (2016). Integración de robótica educativa de bajo coste en el ámbito de la educación secundaria para fomentar el aprendizaje por proyectos. International Journal of Educational Research and Innovation (IJERI), 6, 162-175. http://bit.ly/2ZbzIFG

Vivas, L. \& Sáez, J.M. (2019). Integración de la robótica educativa en Educación Primaria. Revista Latinoamericana de Tecnología Educativa, 18(1), 107-129. https://doi.org/10.17398/1695-288X.18.1.107

Waisburd, G. (2004). El poder de tu creatividad. American Book Store.

Yusuf, J. (2019) Fostering creativity using robotics among gifted primary school students, Gifted and Talented International, 34(1-2), 71-78, https://doi.org/10.1080/15332276.2020.1711545

Zarza, M., \& Holgado, J. (2020). Competencia de pensamiento computacional en la educación no formal. Edutec. Revista Electrónica De Tecnología Educativa, (72), 68-87. https://doi.org/10.21556/edutec.2020.72.1575

\section{INFORMACIÓN SOBRE LA AUTORA}

\section{Paula Morales Almeida \\ Universidad de Las Palmas de Gran Canaria}

Educadora social y psicopedagoga, sus líneas de investigación se centran en la mejora de la calidad de vida de los colectivos más vulnerables: personas sin hogar, familias desestructuradas, infancia y adolescencia en riesgo psicosocial, etc. También se centra en el uso de la robótica y la tecnología educativas en general para favorecer la incorporación de estos colectivos a la sociedad y que tengan las mismas oportunidades. Por último, trabaja a través de los lenguajes artísticos para mejorar las oportunidades de vida de todas estas personas.

Actualmente es profesora ayudante doctora del área de Psicología Evolutiva y de la Educación del Departamento de Psicología, Sociología y Trabajo Social de la Universidad de Las Palmas de Gran Canaria, donde trabaja con familias y colectivos vulnerables.

Los textos publicados en esta revista están sujetos a una licencia de Reconocimiento 4.0 España de Creative Commons. Puede copiarlos, distribuirlos, comunicarlos públicamente y hacer obras derivadas siempre que reconozca los créditos de las obras (autoría, nombre de la revista, institución editora) de la manera especificada por los autores o por la revista. La licencia completa se puede consultar en:Licencia Creative Commons Atribución-NoComercial-Compartir por igual 4.0 Internacional. 